\section{Implicações da doença de Alzheimer na qualidade de vida do cuidador: um estudo comparativo}

\author{
Implications of Alzheimer's disease for the \\ caregiver's quality of life: a comparative study
}

\author{
1 Universidade Federal de São \\ Carlos, São Carlos, Brasil. \\ Correspondência \\ K. Inouye \\ Universidade Federal de São \\ Carlos. \\ Av. Miguel Damha 1000 \\ Unidade 52, São Carlos, SP \\ 13565-814, Brasil. \\ keikain@terra.com.br
}

\begin{abstract}
This study aimed to compare overall and dimension-specific perceptions of quality of life between a group of caregivers of elderly patients with Alzheimer's disease and a group of non-caregivers, matched for sociodemographic variables. Quality of life was measured with the Quality of Life Evaluation Scale in Alzheimer's Disease (Caregiver Version). Statistical analyses were performed (de scriptive and ordinal logistic regression). Caregivers showed significantly higher odds of negatively evaluating the dimensions of physical health, disposition, mood, memory, you in general, and the capacity to perform leisure activities as compared to non-caregivers. Mean total quality of life score was 38.83 for caregivers and 41.81 for noncaregivers. The data suggest that contact with Alzheimer's disease negatively influences the total score and some dimensions of quality of life for family caregivers.
\end{abstract}

Caregivers; Quality of Life; Alzheimer Disease; Dementia
Keika Inouye 1

Elisete Silva Pedrazzani 1

Sofia Cristina Iost Pavarini 1

\section{Introdução}

Atualmente, é quantificável o crescimento do número de idosos que necessitam de tratamento domiciliar e este cuidado envolve familiares, parentes e amigos 1,2. O acompanhamento de um idoso com doença de Alzheimer produz desgastes emocional, psicológico e financeiro para o cuidador familiar, pelo fato de o tratamento ser dispendioso e de o paciente perder gradualmente suas funções cognitivas e evoluir para quadros de total dependência 1 . Essa situação exige redimensionamento da vida dos membros familiares para ser possível conviverem com as implicações causadas pela doença, fatos que conduzem à significativa interferência na qualidade de vida dos mesmos ${ }^{3}$.

Qualidade de vida é uma percepção eminentemente humana que abarca diversos significados derivados de experiências, valores e conhecimentos individuais e coletivos 4 . Atualmente, a definição mais divulgada e conhecida é a do grupo da Organização Mundial da Saúde (OMS) que a descreve como: "a percepção do indivíduo de sua posição na vida, no contexto da cultura e sistemas de valores nos quais vive e em relação aos seus objetivos, expectativas, padrões e preocupações" 5 (p. 1405).

No Brasil, o número de idosos com demência tem crescido rapidamente, mas há carência de dados empíricos sobre o impacto em cuidadores informais ${ }^{6}$. Embora o cuidador familiar tenha 
importância crucial no Brasil, pois as ações do Estado são insuficientes no quadro da saúde pública 7,8; a grande maioria da população de cuidadores familiares ainda não possui as informações e o suporte necessários à assistência e este fato constitui fator de risco para seu desgaste físico, emocional e social 6,9.

Dessa forma, estudos que têm a proposta de verificar até que ponto a doença de Alzheimer tem impacto sobre a qualidade de vida das pessoas envolvidas e quais os possíveis elementos facilitadores e agravantes da situação são considerados um fator de importância crescente 6 . Nesse contexto, o presente estudo teve por objetivo comparar a percepção geral e de cada dimensão de qualidade de vida, de um grupo de cuidadores de idosos com doença de Alzheimer com um grupo de não-cuidadores, emparelhados quanto às variáveis sociodemográficas, a fim de apontar em que extensão esta enfermidade pode afetar a qualidade de vida daqueles que com ela convivem.

\section{Método}

\section{Local e período do estudo}

O presente estudo foi realizado em um município de porte médio, situado na região central do Estado de São Paulo, o qual, segundo estimativa do Instituto Brasileiro de Geografia e Estatística (IBGE), em 2007, tinha uma população de aproximadamente 213 mil habitantes; dos quais $12 \%$ tinham 60 anos de idade ou mais. Pelo fato de nesta pesquisa ter sido encontrado percentual de idosos maior que a média nacional de 8,6\%, é esperado haver maior incidência e prevalência de doenças crônico-degenerativas relacionadas ao envelhecimento ${ }^{10}$, e, por conseqüência, maior número de cuidadores. A coleta de dados teve duração de nove meses, com início em agosto de 2006 e término em abril de 2007.

\section{Participantes}

Este estudo contou com a participação de 106 indivíduos de pesquisa, divididos em dois grupos (n = 53), assim denominados: (a) $\mathrm{G}_{\mathrm{CUID}}$ - grupo composto por cuidadores familiares primários de idosos diagnosticados com doença de Alzheimer atendidos pelo Programa do Medicamento Excepcional; (b) $\mathrm{G}_{\mathrm{NÃO} \text {-CUID }}$ - grupo de comparação composto por não-cuidadores com idade, sexo e status socioeconômico emparelhados ao $\mathrm{G}_{\text {CUID }}$.

\section{Instrumentos}

a) Ficha de Caracterização Sociodemográfica do Participante: teve como finalidade a coleta de dados sociodemográficos do participante, com informações sobre atividades cotidianas e de convívio social, tais como a participação em atividades físicas, grupo religioso, clubes para a terceira idade, tratamento psicológico, fisioterapia e Universidade Aberta à Terceira Idade. Também foram coletados dados de composição familiar, atividades profissionais, tempo para o lazer e/ou hobbies;

b) Questionário Critério Brasil (Associação Nacional de Empresas de Pesquisa. http://www. anep.org.br/codigosguias/CCEB.pdf, acessado em 07/Abr/2008): utilizado para avaliar o nível socioeconômico por meio do poder aquisitivo familiar, com base na quantidade de posse de bens de consumo duráveis, do grau de instrução do chefe da família e da presença de empregada doméstica. Essa escala divide a população em sete classes sociais (A1, A2, B1, B2, C, D e E);

c) Escala de Avaliação da Qualidade de Vida na Doença de Alzheimer - Versão Cuidador (CQdV-DA) 11: esta é uma escala genérica de avaliação de qualidade de vida validada para a cultura brasileira, cujos 13 itens são: saúde física, disposição, humor, moradia, memória, família, casamento, amigos, você em geral, capacidade de fazer tarefas, capacidade de fazer atividades de lazer, dinheiro e vida em geral. As opções de resposta são pontuadas de 1 a 4 , sendo 1 = "ruim", 2 = "regular", 3 = "bom" e 4 = "excelente".

\section{Procedimento de coleta e análise dos dados}

Após aprovação do estudo pelo Comitê de Ética em Pesquisa (CEP) da Universidade Federal de São Carlos (UFSCar) (protocolo $n^{\circ} .221 / 2006$ ) e anuência da Secretaria Municipal de Saúde de São Carlos (SMS) para consultas em seus arquivos e localização dos idosos diagnosticados com doença de Alzheimer atendidos pelo Programa do Medicamento Excepcional (PME), teve início o levantamento de dados, o qual abrangeu os meses de agosto e setembro de 2006. Essa etapa localizou 148 indivíduos com doença de Alzheimer atendidos pelo PME do município. Eles possuíam diagnóstico médico de doença de Alzheimer de gravidade leve ou moderada, segundo os critérios do Manual Diagnóstico e Estatístico de Doenças Mentais - 4a Edição (DSM-IV) e, também, avaliações objetivas - com a aplicação do Mini-Exame do Estado Mental, cujos escores variavam entre 12 e 24 pontos para pacientes com mais de quatro anos de escolaridade, e entre $8 \mathrm{e}$ 17 pontos para pacientes com até quatro anos de 
estudo, além de pontuações entre 1 e 2 na Escala Clínica de Avaliação de Demência (Clinical Dementia Rating Scale-CDR). Com base nos dados dos sujeitos com doença de Alzheimer, foi iniciada a busca pelos respectivos cuidadores familiares, a qual foi realizada por ordem de sorteio. Para isso, os nomes de todos os indivíduos atendidos pelo PME foram listados, por ordem alfabética, numerados e, então, sorteados pelo número. Os 53 primeiros cuidadores primários concordantes constituíram o grupo $\mathrm{G}_{\text {CUID }}$ e representaram $35,1 \%$ do número total de todos os possíveis cuidadores $(\mathrm{n}=148)$. Alguns dos sujeitos sorteados ( $\mathrm{n}=26)$ não puderam fazer parte de nosso banco por recusa, não localização, falecimento, mudança ou não cumprimento dos critérios de inclusão. São eles:

- Cuidador profissional, e não um familiar coresidente $(\mathrm{n}=7)$;

- Idosos e respectivos cuidadores que não foram encontrados nos endereços cadastrados nas fichas da SMS $(n=6)$;

- Cuidadores de pessoas com diagnóstico de doença de Alzheimer, mas, idade inferior a 60 anos ( $\mathrm{n}=3)$;

- Cuidadores de idosos com distúrbios graves de linguagem e suspeita de estágio grave da doença $(n=5)$;

- Idosos que haviam falecido no intervalo entre o levantamento e a visita da pesquisadora $(\mathrm{n}=2)$;

- Idosos que haviam mudado para outros municípios (para dividir a responsabilidade dos cuidados entre familiares) $(\mathrm{n}=2)$; e

- Não aceitou participar $(n=1)$.

A constituição do $\mathrm{G}_{\mathrm{NÃO}-\mathrm{CUID}}$ foi feita por emparelhamento conforme já descrito no subitem Participantes. Para facilitar a localização dos potenciais sujeitos do $\mathrm{G}_{\mathrm{NÃO} \text {-CUID, }}$ eles podiam ser indicados pelos próprios sujeitos do $\mathrm{G}_{\mathrm{CUID}}$. No entanto, para fazer parte do banco de dados era necessário apresentar as variáveis idade, sexo e status socioeconômico emparelhadas ao $\mathrm{G}_{\text {CUID }}$. Caso o sujeito indicado não atendesse a esses critérios, a pesquisadora entrevistava outros indivíduos até localizar quem satisfizesse os referidos quesitos. Nesse grupo não houve recusas de participação.

Todos os dados coletados foram digitados em banco nos programas SPSS 10.0 (SPSS Inc., Chicago, Estados Unidos) e Stata 8.0 (Stata Corp., College Station, Estados Unidos), para realização de análises estatísticas: (a) descritivas para caracterizar o perfil dos grupos; (b) t de Student e qui-quadrado de Pearson para testar emparelhamento de grupos; e (c) regressão logística ordinal para identificar as diferenças de percepção geral e de cada dimensão de qualidade de vida do grupo de cuidadores familiares e do grupo de não-cuidadores.

\section{Resultados}

\section{Perfil sociodemográfico da amostra de cuidadores familiares de idosos com doença de Alzheimer ( $G_{\text {CUID }}$ )}

Dos 53 componentes do $\mathrm{G}_{\text {CUID }}$, $68 \%$ eram do sexo feminino $(n=36)$ e $32 \%$ do masculino $(n=17)$. A média das idades de ambos os gêneros foi de 63,81 anos (DP: 13,41, $x_{\text {mín }}=37$, $x_{\text {máx }}=89$ ); as mulheres apresentaram uma média de 60,14 anos (DP: $12,21, x_{\text {mín }}=37, x_{\text {máx }}=82$ ) e os homens, de 71,59 anos (DP: 12,80, $x_{\text {mín }}=40, x_{\text {máx }}=89$ ).

Quanto ao grau de parentesco dos cuidadores familiares, a distribuição está apresentada na Tabela 1.

O tempo médio de cuidador era de 3,43 anos (DP: 2,72, $x_{\text {mín }}=0,5, x_{\text {máx }}=16$ ). Quanto à composição familiar, $6 \%(n=3)$ eram compostas por solteiros sem filhos; $4 \%(n=2)$ por casados sem filhos, $4 \%(n=2)$ por separados sem filhos, $4 \%$ $(\mathrm{n}=2)$ separados com filhos, $6 \%$ viúvos com filhos e $77 \%(n=41)$ casados(as) com filhos.

Em relação às atividades ocupacionais, a maioria, 72\% (n = 38), não tinha ocupação fora do lar e informou dedicar-se apenas a cuidar do idoso, $15 \%(n=8)$ trabalhavam apenas meio período e $13 \%(n=7)$ trabalhavam em período integral.

Os dados referentes ao status socioeconômico, obtido pelo Critério de Classificação Econô-

Tabela 1

Distribuição do grau de parentesco do cuidador familiar segundo gênero.

\begin{tabular}{lcc}
\hline Grau de parentesco/Sexo & $\mathbf{n}$ & $\%$ \\
\hline Cônjuge & & \\
$\quad$ Feminino (esposa) & 15 & 28 \\
$\quad$ Masculino (marido) & 13 & 25 \\
Filho & 19 & 36 \\
Feminino (filha) & 4 & 8 \\
Masculino (filho) & 1 & \\
Nora/Genro & 0 & 0 \\
Feminino (nora) & & \\
Masculino (genro) & 1 & 0 \\
Irmão & 0 & 100 \\
Feminino (irmã) & 53 &
\end{tabular}


mica Brasil, revelaram que $8 \%(\mathrm{n}=4)$ dos participantes eram da classe A2; 15\% $(n=8)$ da B1; $15 \%$ $(\mathrm{n}=8)$ da B2; 38\% $(\mathrm{n}=20)$ da C; $23 \%(\mathrm{n}=12)$ da D; e $2 \%(n=1)$ da E.

No que se refere à escolaridade, $30 \%(n=16)$ eram analfabetos ou não haviam concluído a primeira metade do curso fundamental (antigamente denominado primário); $30 \%(n=16)$ não complementaram o curso fundamental, mas tinham primário completo (os quatro primeiros anos do fundamental) ou ginásio incompleto (os últimos quatro anos do fundamental); $6 \%$ $(\mathrm{n}=3)$ ginásio completo (Ensino Fundamental) ou colegial incompleto (Ensino Médio), 15\% ( $\mathrm{n}=8$ ) colegial completo (Ensino Médio) ou superior incompleto, e 19\% ( $\mathrm{n}=10)$ tinham superior completo. A nomenclatura que classifica a escolaridade no momento do estudo não é a oficialmente utilizada. Entretanto, ela foi escolhida para por ser mais familiar para os participantes, além de ser a que consta no Critério de Classificação Econômica Brasil. Para correspondência, a educação básica atual abrange: ensino infantil = pré-escola; Ensino Fundamental = primário + ginásio; Ensino Médio = colegial .

Quanto às práticas cotidianas, 47\% $(n=25)$ dos cuidadores familiares dedicavam pelo menos uma hora da semana para alguma atividade física. Quando questionados sobre que fatores estariam relacionados ao melhor enfrentamento das adversidades inerentes à doença de Alzheimer em sua vida, os familiares contavam principalmente com uma pessoa para dividir a tarefa de cuidar em finais de semana e eventuais necessidades $(36 \% \mathrm{n}=19)$, encontravam conforto e amparo na religião $(23 \%, \mathrm{n}=12)$. Entre os demais, 3 (6\%) indivíduos haviam sido capacitados para serem cuidadores; 2 (4\%) estavam em tratamento psicológico; 2 (4\%) tinham hobbies; 1 (2\%) freqüentava a Universidade Aberta à Terceira Idade; e 14 (26\%) disseram não dispor de qualquer auxílio, apoio ou oportunidades de lazer.

\section{Perfil sociodemográfico da amostra de não-cuidadores ( $G_{\text {NÃO-CUID }}$ )}

A composição do $G_{\text {NÃO-CUID }}$, quanto à variável gênero, é idêntica à do $\mathrm{G}_{\text {CUID }}(68 \%, \mathrm{n}=36$, do gênero feminino; e $32 \%, \mathrm{n}=17$, do gênero masculino). A média das idades desse grupo para ambos os gêneros era de 63,55 anos (DP: 13,22, $x_{\text {mín }}=$ $35, x_{\text {máx }}=88$ ), as mulheres apresentaram média de 60,11 anos (DP: 12,36, $x_{\text {mín }}=35, x_{\text {máx }}=82$ ) e os homens, de 70,82 anos (DP: 12,29, $x_{\text {mín }}=44$, $\mathrm{x}_{\text {máx }}=88$ ).

A tendência predominante de analfabetismo acompanhou o grupo, $42 \%(n=22)$ dos componentes eram analfabetos ou tinham apenas o primário incompleto; $25 \%(\mathrm{n}=13)$ tinham primário completo ou ginásio incompleto; $11 \%$ (n = 6) ginásio completo ou colegial incompleto; $13 \%$ $(n=7)$ colegial completo ou superior incompleto; e $9 \%(n=5)$ tinham curso superior completo.

No que se refere à distribuição socioeconômica, $2 \%(n=1)$ dos participantes pertenciam à classe $\mathrm{A} 1 ; 6 \%(\mathrm{n}=3)$ à $\mathrm{B} 1 ; 11 \%(\mathrm{n}=6)$ à $\mathrm{B} 2 ; 45 \%$ à $\mathrm{C} ; \mathrm{e} 36 \%(\mathrm{n}=19)$ à classe $\mathrm{D}$.

A composição familiar mais freqüente do $\mathrm{G}_{\mathrm{NÃO}-\mathrm{CUID}}$ era de indivíduos casados com filhos $(58 \%, \mathrm{n}=31)$, seguida de viúvos com filhos $(21 \%$, $\mathrm{n}=11$ ), e foram encontradas proporções iguais de sujeitos separados com filhos e solteiros sem filhos $(9 \%, \mathrm{n}=5)$.

Em relação às atividades ocupacionais, devido à idade elevada de alguns componentes, a maioria, 60\% ( $\mathrm{n}=32$ ), não trabalhava fora por já estar aposentado(a) ou porque tinha parado de trabalhar por razões diversas; $13 \%(\mathrm{n}=7)$ trabalhavam apenas meio período; e 26\% ( $n=14)$ trabalhavam em período integral.

Quanto à prática de exercícios físicos, $60 \%$ ( $\mathrm{n}=32$ ) do $\mathrm{G}_{\text {NÃO-CUID }}$ dedicavam pelo menos uma hora da semana à caminhada $(\mathrm{n}=24,45 \%)$, ginástica em academia $(n=3,6 \%)$, dança $(n=2$, $4 \%)$, natação $(\mathrm{n}=1,2 \%)$, hidroginástica $(\mathrm{n}=1$, $2 \%)$ ou ginástica terapêutica $(n=1,2 \%)$. No que se refere às atividades otimizadoras da qualidade de vida, o grupo dividiu-se em $49 \%(\mathrm{n}=26)$ que acreditavam não dispor contra $51 \%(\mathrm{n}=27)$ que percebiam o grupo religioso ou hobbies como fontes de bem-estar.

\section{Teste de emparelhamento do $\mathrm{G}_{\text {CUID }}$ e \\ $\mathrm{G}_{\text {Não-CUID }}$ quanto às variáveis sociodemográficas e atividades cotidianas}

Os grupos $\mathrm{G}_{\text {CUID }}$ e $\mathrm{G}_{\mathrm{NÃO} \text {-CUID, }}$ estatisticamente, não diferiam quanto às variáveis idade, sexo, composição familiar, ocupação laboral, status socioeconômico, nível de instrução e prática de atividades físicas conforme os dados apresentados na Tabela 2 .

\section{Avaliação comparativa entre cada dimensão da qualidade de vida e o escore total obtido na CQdV-DA dos grupos $\mathrm{G}_{\text {CUID }}$ e $\mathrm{G}_{\text {NÃO-CUID }}$}

Os resultados da avaliação de cada dimensão de qualidade de vida obtidos por meio da CQdV-DA, bem como a significância das análises de regressão logística ordinal dos grupos $\mathrm{G}_{\text {CUID }} \mathrm{e}$ $\mathrm{G}_{\text {NÃO-CUID }}$ estão expressos na Tabela 3.

Embora todos os 13 itens de qualidade de vida testados apontassem melhor percepção do grupo de não-cuidadores, sete itens não apresentaram chance significativamente maior de serem 
Tabela 2

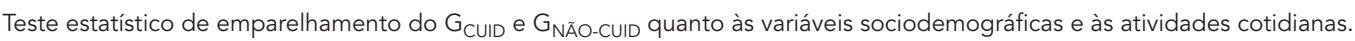

\begin{tabular}{|c|c|c|c|}
\hline Variáveis & $\mathrm{G}_{\text {CUID }}$ & $\mathrm{G}_{\mathrm{NÃO}-\mathrm{CUID}}$ & Análise estatística comparativa \\
\hline \multirow[t]{2}{*}{ Idade (anos) [média] } & 63,81 (DP: 13,41; & 63,55 (DP: 13,22; & $t(104)=0,102$ \\
\hline & $\left.x_{\min }=37, x_{\operatorname{máx}}=89\right)$ & $\left.x_{\min }=35, x_{\text {máx }}=88\right)$ & $p=0,919$ * \\
\hline \multirow[t]{2}{*}{ Tempo de cuidador (anos) [média] } & 3,43 (DP: 2,$72 ;$ & - & $t(52)=9,176$ \\
\hline & $\left.x_{\min }=0,5, x_{\operatorname{máx}}=16\right)$ & & $p=0,000 * \star$ \\
\hline \multicolumn{4}{|l|}{ Sexo } \\
\hline Feminino & $36(68 \%)$ & $36(68 \%)$ & $\chi^{2}(1)=0,0$ \\
\hline Masculino & $17(32 \%)$ & 17 (32\%) & $p=1,000$ * \\
\hline \multicolumn{4}{|l|}{ Composição familiar } \\
\hline Separados sem filhos & $2(4 \%)$ & $0(0 \%)$ & $\chi^{2}(5)=10,079$ \\
\hline Separados com filhos & $2(4 \%)$ & $5(9 \%)$ & $p=0,073$ * \\
\hline Viúvos com filhos & $3(6 \%)$ & $11(21 \%)$ & \\
\hline Solteiros sem filhos & $3(6 \%)$ & $5(9 \%)$ & \\
\hline Casados sem filhos & $2(4 \%)$ & $0(0 \%)$ & \\
\hline Casados com filhos & 41 (77\%) & 31 (58\%) & \\
\hline \multicolumn{4}{|l|}{ Ocupação laboral } \\
\hline Não trabalha fora & 38 (72\%) & $32(60 \%)$ & $\chi^{2}(6)=8-527$ \\
\hline Trabalha $1 / 2$ período & $8(15 \%)$ & $7(13 \%)$ & $p=0,202$ * \\
\hline Trabalha em período integral & $7(13 \%)$ & $14(26 \%)$ & \\
\hline \multicolumn{4}{|l|}{ Status socioeconômico } \\
\hline $\mathrm{A} 1$ & $0(0 \%)$ & $1(2 \%)$ & $\chi^{2}(5)=7,303$ \\
\hline $\mathrm{A} 2$ & $4(8 \%)$ & $0(0 \%)$ & $p=0,199$ * \\
\hline B1 & $8(15 \%)$ & $3(6 \%)$ & \\
\hline B2 & $8(15 \%)$ & $6(11 \%)$ & \\
\hline C & $20(38 \%)$ & $24(45 \%)$ & \\
\hline $\mathrm{D}$ & $12(23 \%)$ & 19 (36\%) & \\
\hline E & $1(2 \%)$ & $0(0 \%)$ & \\
\hline \multicolumn{4}{|l|}{ Nível de instrução } \\
\hline Analfabeto ou primário incompleto & $16(30 \%)$ & $22(42 \%)$ & $\chi^{2}(4)=3,991$ \\
\hline Primário completo ou ginásio incompleto & $16(30 \%)$ & $13(25 \%)$ & $p=0,407$ * \\
\hline Ginásio completo ou colegial incompleto & $3(6 \%)$ & $6(11 \%)$ & \\
\hline Colegial completo ou superior incompleto & $8(15 \%)$ & $7(13 \%)$ & \\
\hline Superior completo & 10 (19\%) & 5 (9\%) & \\
\hline \multicolumn{4}{|l|}{ Atividade física } \\
\hline Pratica & $25(47 \%)$ & $32(60 \%)$ & $\chi^{2}(6)=4,244$ \\
\hline Não pratica & $28(53 \%)$ & $21(40 \%)$ & $p=0,644$ * \\
\hline
\end{tabular}

$\mathrm{G}_{\text {CUID }}$ : grupo composto por cuidadores familiares primários de idosos diagnosticados com doença de Alzheimer atendidos pelo Programa do Medicamento

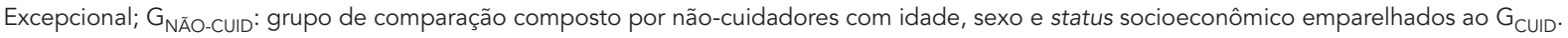

* Não significante;

** $p<0,001$.

avaliados negativamente; a saber: moradia, família, casamento, amigos, capacidade de fazer tarefas, dinheiro e a vida em geral. Os itens de qualidade de vida que apresentavam razão desfavorável para o $\mathrm{G}_{\mathrm{CUID}}$ foram seis: saúde física, disposição, humor, memória, você em geral e capacidade para fazer atividades de lazer.
Quanto aos escores totais de qualidade de vida, foi encontrada a média de 38,83 pontos para cuidadores e de 41,81 para não-cuidadores (RC: $3,05, p=0,003)$. A Figura 1 mostra a distribuição do número de sujeitos de cada grupo segundo os escores totais obtidos por meio da CQdV-DA; nela vale observar o deslocamento do $\mathrm{G}_{\mathrm{NÃO} \text {-CUID }}$ para pontuações superiores (à direita). 
Tabela 3

Comparativo da avaliação de cada dimensão da qualidade de vida e significância das análises de regressão logística ordinal dos grupos de cuidadores familiares ( $\left.\mathrm{G}_{\mathrm{CUID}}\right)$ e não-cuidadores ( $\left.\mathrm{G}_{\text {NÃO-CUID }}\right)$.

\begin{tabular}{|c|c|c|c|c|c|c|c|}
\hline \multirow[t]{2}{*}{ Dimensões/Grupo } & \multicolumn{4}{|c|}{ Percepção dos grupos $\mathrm{G}_{\text {CUID }}$ e $\mathrm{G}_{\text {NÃO-CUID }}$} & \multirow{2}{*}{$\begin{array}{l}\text { Razão de } \\
\text { chances }\end{array}$} & \multirow[t]{2}{*}{ IC95\% } & \multirow[t]{2}{*}{ Valor de $\mathrm{p}$} \\
\hline & $\begin{array}{l}\text { Ruim } \\
\text { n (\%) }\end{array}$ & $\begin{array}{c}\text { Regular } \\
\text { n (\%) }\end{array}$ & $\begin{array}{l}\text { Bom } \\
\text { n (\%) }\end{array}$ & $\begin{array}{c}\text { Excelente } \\
\text { n (\%) }\end{array}$ & & & \\
\hline \multicolumn{8}{|l|}{ 1. Saúde física } \\
\hline $\mathrm{G}_{\mathrm{CUID}}$ & $2(4)$ & $20(38)$ & $23(43)$ & $8(15)$ & 2,48 & $1,19-5,18$ & 0,015 * \\
\hline $\mathrm{C}_{\mathrm{NÄO} O-C U I D}$ & $1(2)$ & $10(19)$ & $27(51)$ & $15(28)$ & & & \\
\hline \multicolumn{8}{|l|}{ 2. Disposição } \\
\hline $\mathrm{G}_{\text {CUID }}$ & $0(0)$ & $11(21)$ & $30(57)$ & $12(23)$ & 2,27 & $1,08-4,78$ & 0,030 * \\
\hline $\mathrm{C}_{\mathrm{NÄO}-\mathrm{CUID}}$ & $0(0)$ & $7(13)$ & $23(43)$ & $23(43)$ & & & \\
\hline \multicolumn{8}{|l|}{ 3. Humor } \\
\hline $\mathrm{G}_{\text {CUID }}$ & $1(2)$ & $10(19)$ & $30(57)$ & $12(23)$ & 3,05 & $1,39-6,69$ & $0,005 * \star$ \\
\hline $\mathrm{C}_{\mathrm{NÄO}-\mathrm{CUID}}$ & $0(0)$ & $2(4)$ & $29(55)$ & $22(42)$ & & & \\
\hline \multicolumn{8}{|l|}{ 4. Moradia } \\
\hline $\mathrm{G}_{\text {CUID }}$ & $0(0)$ & $7(13)$ & $36(68)$ & $10(19)$ & 1,26 & $0,52-3,10$ & $0,607 * \star *$ \\
\hline $\mathrm{C}_{\mathrm{NÄO}-\mathrm{CUID}}$ & $0(0)$ & $1(2)$ & $45(85)$ & $7(13)$ & & & \\
\hline \multicolumn{8}{|l|}{ 5. Memória } \\
\hline $\mathrm{G}_{\text {CUID }}$ & $0(0)$ & $6(11)$ & $23(43)$ & $24(45)$ & 3,06 & $1,38-6,78$ & $0,006 \#$ \\
\hline$C_{\text {NÃO-CUID }}$ & $0(0)$ & $4(8)$ & $10(19)$ & $39(74)$ & & & \\
\hline \multicolumn{8}{|l|}{ 6. Família } \\
\hline $\mathrm{G}_{\text {CUID }}$ & $0(0)$ & $0(0)$ & $30(57)$ & $23(43)$ & 1,58 & $0,73-3,39$ & $0,245 * \star \star$ \\
\hline$C_{\text {NÃO-CUID }}$ & $0(0)$ & $0(0)$ & $24(45)$ & $29(55)$ & & & \\
\hline \multicolumn{8}{|l|}{ 7. Casamento } \\
\hline $\mathrm{G}_{\mathrm{CUID}}$ & $0(0)$ & $2(4)$ & $25(47)$ & $26(49)$ & 1,67 & $0,78-3,04$ & $0,189 * \star *$ \\
\hline$C_{\text {NÃO-CUID }}$ & $0(0)$ & $0(0)$ & $21(40)$ & $32(60)$ & & & \\
\hline \multicolumn{8}{|l|}{ 8. Amigos } \\
\hline $\mathrm{G}_{\text {CUID }}$ & $0(0)$ & $4(8)$ & $25(47)$ & $24(45)$ & 1,44 & $0,68-3,05$ & $0,344 * \star \star$ \\
\hline $\mathrm{C}_{\mathrm{NÄO}-\mathrm{CUID}}$ & $0(0)$ & $0(0)$ & $26(49)$ & $27(51)$ & & & \\
\hline \multicolumn{8}{|l|}{ 9. Você em geral } \\
\hline $\mathrm{G}_{\text {CUID }}$ & $0(0)$ & $11(21)$ & $29(55)$ & $13(25)$ & 2,21 & $1,03-4,76$ & 0,042 * \\
\hline$C_{\text {NÃO-CUID }}$ & $0(0)$ & $3(6)$ & $31(58)$ & $19(36)$ & & & \\
\hline \multicolumn{8}{|l|}{ 10. Tarefas } \\
\hline $\mathrm{G}_{\text {CUID }}$ & $0(0)$ & $5(9)$ & $27(51)$ & $21(40)$ & 1,47 & $0,70-3,09$ & $0,306 * \star *$ \\
\hline$C_{\text {NÃO-CUID }}$ & $3(6)$ & $0(0)$ & $24(45)$ & $26(49)$ & & & \\
\hline \multicolumn{8}{|l|}{ 11. Lazer } \\
\hline $\mathrm{G}_{\text {CUID }}$ & $2(4)$ & $13(25)$ & $25(47)$ & $13(25)$ & 2,26 & $1,08-4,71$ & 0,030 * \\
\hline $\mathrm{G}_{\mathrm{CUID}}$ & $3(6)$ & $3(6)$ & $26(49)$ & $21(40)$ & & & \\
\hline \multicolumn{8}{|l|}{ 12. Dinheiro } \\
\hline $\mathrm{G}_{\text {CUID }}$ & $11(21)$ & $35(66)$ & $7(13)$ & $0(0)$ & 2,12 & $0,88-5,08$ & 0,094 *** \\
\hline$C_{\text {NÃO-CUID }}$ & $3(6)$ & 42 (79) & $7(13)$ & $1(2)$ & & & \\
\hline \multicolumn{8}{|l|}{ 13. Vida geral } \\
\hline $\mathrm{G}_{\mathrm{CUID}}$ & $1(2)$ & $21(40)$ & $24(45)$ & $7(13)$ & 2,04 & $0,92-4,53$ & 0,081 *** \\
\hline $\mathrm{C}_{\mathrm{NÄO}-\mathrm{CUID}}$ & $0(0)$ & $8(15)$ & 44 (83) & $1(2)$ & & & \\
\hline
\end{tabular}

* $p<0,05$;

** $p \leq 0,005$

*** Não significante;

$\# p<0,01$. 
Distribuição do número de sujeitos dos grupos de cuidadores e não-cuidadores segundo as pontuações finais obtidas por meio da Escala de Avaliação da Qualidade de Vida na Doença de Alzheimer - Versão Cuidador (CQdV-DA).
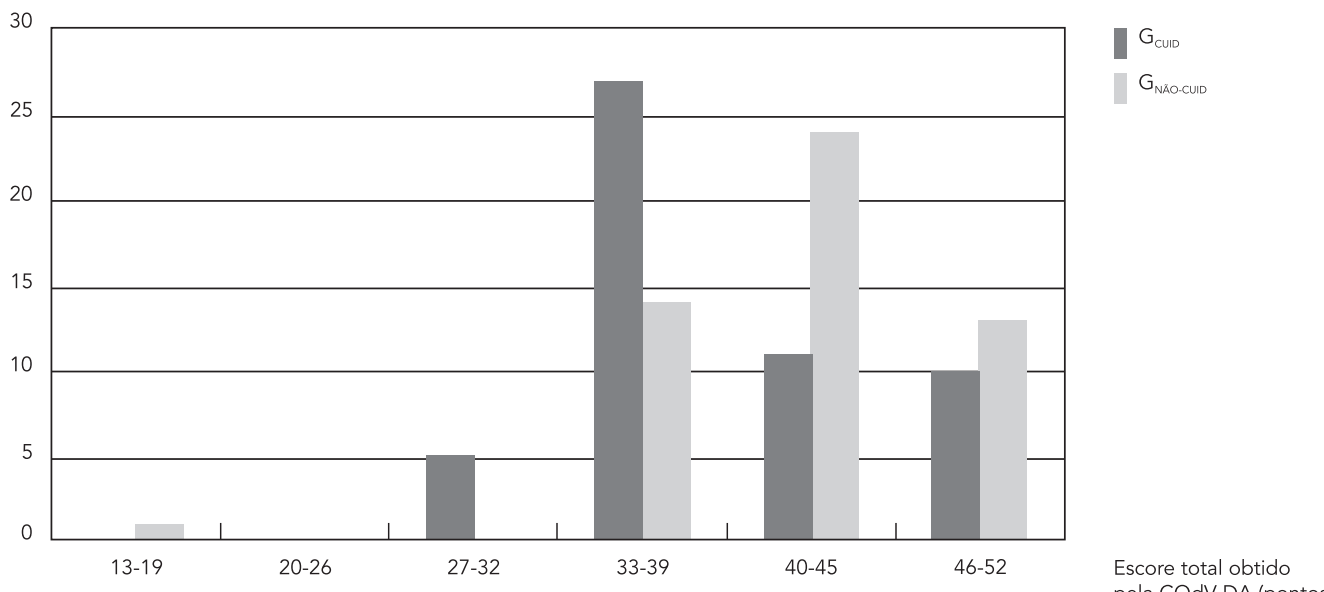

\section{Discussão}

No Brasil, por causas predominantemente culturais, o papel da mulher cuidadora ainda é uma atribuição esperada pela sociedade, por ser ela a principal agente social na dinâmica dos cuidados pessoais necessários às atividades de vida diária dos idosos com doença de Alzheimer 2,6,8,12. Em $68 \%(\mathrm{n}=36)$ dos casos pesquisados, o cuidador tinha o perfil típico descrito na literatura: mulheres, residentes no mesmo domicílio, geralmente filhas ou esposas $(28 \%, n=15)$. No entanto, a participação dos homens também foi expressiva (32\%), mas cabe alertar para a importância de serem consideradas as questões de gênero do cuidador no planejamento de intervenções psicoeducacionais. Outra importante consideração deste trabalho é o alto percentual (64\%) de familiares com 60 anos ou mais a exercerem o papel de cuidador, o que corrobora dados de outros estudos que apontam para um número significativo de pessoas idosas que cuidam de idosos 12 .

A média de idade elevada dos cuidadores familiares ( $\mathrm{M}=63,81$ anos) explica o número majoritário de participantes que já não trabalhavam no momento da entrevista. Os familiares que trabalhavam em período integral eram responsáveis por casos menos graves da doença de Alzheimer que, apesar dos problemas de memória, mantinham relativa autonomia. Isso permite inferir que casos moderados da doença de Alzheimer exigem dedicação maior e, muitas vezes, exclusiva por parte do responsável.

Visto que no município em que a pesquisa foi realizada não existe serviço especializado em diagnóstico diferencial das fases da doença, este estudo limitou-se à análise da qualidade de vida de cuidadores de idosos com doença de Alzheimer provável de gravidade leve e moderada, pois apenas estes eram atendidos no PME, no qual foram buscados os primeiros dados para ser possível alcançar os cuidadores. Os familiares responsáveis por casos severos da doença não foram contemplados nesta investigação, portanto, será interessante um trabalho a ser replicado em grandes centros, dada a possibilidade de, nestes casos, ser encontrado maior número de itens de qualidade de vida afetados pelas demandas de cuidado.

No que diz respeito às atividades cotidianas, percebemos que o grupo de não- cuidadores era mais ativo, principalmente quanto à: ocupação; atividades físicas, religiosas e de convívio social. Embora não seja possível afirmar que essa característica eleve sua qualidade de vida, podemos apontar na direção contrária - que os cuidadores têm vivido em condições adversas que prejudicam suas atividades de convívio social.

Dos 13 itens de qualidade de vida testados, os seis significantemente desfavoráveis para o cuidador (saúde física, disposição, humor, você em geral, capacidade de fazer atividades de lazer 
e memória) possivelmente estão relacionados à sobrecarga que limita o cotidiano do familiar, inclusive nos cuidados com o próprio bem-estar e saúde. Quanto à "memória", cabe ressalvar que muitos familiares, por conviverem com pessoas com prejuízo de memória, tendem a estabelecer padrões mais rigorosos desta variável por conhecerem de perto os problemas que sua falta acarreta na vida do indivíduo e pelo receio de que esta situação possa se estabelecer em sua vida.

Embora a maioria dos estudos foque apenas o impacto negativo de ser cuidador, os fatores positivos podem ajudar no desenvolvimento de estratégias de enfrentamento 13. Dessa forma, os sete itens de qualidade de vida não desfavoráveis para o $\mathrm{G}_{\text {CUID }}$ (moradia, família, casamento, amigos, capacidade de fazer tarefas, dinheiro e a vida em geral) apontados neste estudo não podem ser ignorados. Isoladas as percepções positivas (bom e excelente) das percepções negativas (ruim e regular), é possível verificar que o $\mathrm{G}_{\text {CUID }}$ encontrava maior grau de satisfação nos relacionamentos interpessoais. Todos os cuidadores $(100 \%, \mathrm{n}=53)$ consideraram suas famílias boas ou excelentes, $96 \%$ ( $n=51)$ o casamento ou relacionamento próximo e $92 \%(n=49)$ as amizades.

\section{Conclusões}

Uma vez que os resultados apontaram para o fato de algumas vezes haver influência desfavorável na convivência com a doença de Alzheimer na percepção de qualidade de vida do cuidador familiar, é preciso insistir que as intervenções sejam voltadas para ambos. O cuidador é uma importante fonte de apoio para o enfrentamento da dependência imposta pela demência, sendo assim, o estímulo e o fortalecimento de parcerias entre cuidadores familiares e profissionais pode minimizar as dificuldades vivenciadas 14 . Por meio de esforços conjuntos será possível pensar em medidas preventivas e intervenções que garantam novos sentimentos que estejam livres de estereótipos associados ao papel de cuidar.

Pelo fato de a incidência de doenças crônicas crescer proporcionalmente ao envelhecimento, também deverá aumentar proporcionalmente o número de pessoas que se tornem cuidadores. Dentro dessa perspectiva, a preocupação com o desenvolvimento de práticas de ações, que atendam às dimensões de qualidade de vida percebidas com mais pessimismo e fortaleçam as demais, reúne-se em torno de uma significação inclusiva, flexível e dinâmica que leve em conta o valor da opinião do próprio cuidador. As intervenções psicoeducacionais devem apoiar a otimização de conhecimentos e aptidões que proporcionem encorajamento e satisfaçam as necessidades individuais, para que os familiares se sintam aceitos e compreendidos em suas dificuldades e limitações.

As percepções e principais dificuldades apontadas pelos cuidadores podem contribuir para que seja fortalecido o processo de zelo com a qualidade de vida do familiar. O comprometimento com a promoção da saúde, em suas perspectivas mais amplas, deve abarcar objetivos que ultrapassem a visão simplista de acesso a consultas médicas e medicamentos. A qualidade de vida tem caráter mais abrangente e reconhece a importância da participação em relações sociais, econômicas, culturais, espirituais e civis por meio de um processo contínuo de educação, crescimento, atualização e realização pessoal que os permita continuar como membros ativos e construtores da sociedade.

Identificar o perfil dessa população, bem como suas especificidades, possibilita intervenções direcionadas que poderão contribuir para a obtenção de melhoria na qualidade de vida dos indivíduos e até mesmo levar à redução de gastos públicos com cuidados. Integrar preservação e melhoria da sensação de bem-estar de todos os coletivos deve ser alvo de reflexão, questionamentos, discussões e planos de ação em todas as esferas para que as últimas etapas da vida sejam acompanhadas de satisfação tanto para o idoso como para aqueles que o cercam.

Ainda que este estudo apresente limitações metodológicas - como tamanho da amostra relativamente pequeno e a pontualidade da experiência que foi realizada em uma única cidade do interior paulista - esta investigação é parte do conjunto de trabalhos que contribui para ampliar os conhecimentos sobre o impacto de ser cuidador na vida de um familiar. Por esse motivo, parece interessante que venham a ser feitas investigações semelhantes, em outras realidades, com um número maior de participantes; e que também sejam levadas a efeito pesquisas que correlacionem a qualidade de vida do cuidador às características do paciente, tais como grau de severidade da doença e de problemas psicológicos (depressão, agressividade e ansiedade). Também seria importante que fossem realizados estudos sobre os recursos acessíveis e o ambiente familiar do cuidador. Os resultados de tais investigações permitiriam abordar e planejar programas sociais que atendessem as principais necessidades dos envolvidos nesse status quo. 


\section{Resumo}

Este estudo teve como objetivo comparar a percepção geral e de cada dimensão de qualidade de vida de um grupo de cuidadores de idosos com doença de Alzheimer com um grupo de não-cuidadores emparelhado quanto às variáveis sociodemográficas. As medidas de qualidade de vida foram obtidas por meio da Escala de Avaliação da Qualidade de Vida na Doença de Alzheimer - Versão Cuidador. Foram realizadas análises estatísticas (descritivas e regressão logística ordinal). O grupo de cuidadores apresentou chance maior e significativa de avaliar negativamente as dimensões saúde física, disposição, humor, memória, você em geral e capacidade para fazer atividades de lazer quando comparado ao grupo de não-cuidadores. Quanto aos escores totais de qualidade de vida, a média obtida foi de 38,83 pontos para cuidadores e de 41,81 para nãocuidadores. Esses dados sugerem que o convívio com a doença de Alzheimer influencia negativamente o escore total e algumas dimensões de qualidade de vida do cuidador familiar.

Cuidadores; Qualidade de Vida; Doença de Alzheimer; Demência

\section{Referências}

1. Luzardo AR, Gorini MIPC, Silva APSS. Características de idosos com doença de Alzheimer e seus cuidadores: uma série de casos em um serviço de neurogeriatria. Texto \& Contexto Enferm 2006; 15:587-94.

2. Fernandes MGM, Garcia TR. Determinantes da tensão do cuidador familiar de idosos dependentes. Rev Bras Enferm 2009; 62:393-9.

3. Caldas CP. Envelhecimento com dependência: responsabilidades e demandas da família. Cad Saúde Pública 2003; 19:733-81.

4. Dantas RAS, Sawada NO, Malerbo MB. Pesquisas sobre qualidade de vida: revisão da produção científica das universidades públicas do Estado de São Paulo. Rev Latinoam Enferm 2003; 11:532-8.

5. World Health Organization Quality of Life Group. The World Health Organization Quality of Life assessment (WHOQOL): position paper from the World Health Organization. Soc Sci Med 1995; 41:1403-9

6. Garrido R, Menezes PR. Impacto em cuidadores de idosos com demência atendidos em um serviço psicogeriátrico. Rev Saúde Pública 2004; 38 : 835-41.

7. Pitta AMF. A equação humana no cuidado à doença: o doente, seu cuidador e as organizações de saúde. Saúde Soc 1996; 5:35-60.

8. Silveira TM, Caldas CP, Carneiro TF. Cuidando de idosos altamente dependentes na comunidade: um estudo sobre cuidadores familiares principais. Cad Saúde Pública 2006; 22:1629-38.

\section{Colaboradores}

K. Inouye, E. S. Pedrazzani e S. C. I. Pavarini participaram de todas as etapas da elaboração do artigo: concepção e projeto, análise e interpretação dos dados, redação, revisão crítica relevante do conteúdo intelectual e aprovação final.

9. Cerqueira ATAR, Oliveira NIL. Programa de apoio a cuidadores: uma ação terapêutica e preventiva na atenção à saúde dos idosos. Psicol USP 2002; 13:133-50

10. Instituto Brasileiro de Geografia e Estatística. População recenseada e estimada, segundo os municípios de São Paulo em 2007. http://ibge.gov.br/ home/estatistica/populacao/contagem 2007/con tagem_final/tabela1_1_20.pdf (acessado em 08/ Abr/2010).

11. Novelli MMPC, Dal Rovere HH, Nitrini R, Caramelli P. Adaptação transcultural da escala de avaliação da qualidade de vida na doença de Alzheimer. Arq Neuropsiquiatr 2005, 63:201-6.

12. Karsch UM. Idosos dependentes: famílias e cuidadores. Cad Saúde Pública 2003; 19:861-6.

13. Laham CF. Percepção de perdas e ganhos subjetivos entre cuidadores de pacientes atendidos em um programa de assistência domiciliar [Dissertação de Mestrado]. São Paulo: Faculdade de Medicina, Universidade de São Paulo; 2003.

14. De Paula JA, Roque FP, Araújo FS. Qualidade de vida em cuidadores de idosos portadores de demência de Alzheimer. J Bras Psiquiatr 2008; 57:283-7.

Recebido em 15/Out/2009

Versão final reapresentada em 18/Mar/2010

Aprovação em 01/Abr/2010 